\title{
Combined Effects of Hall Current and Radiation on MHD Free Convective Flow in a Vertical Channel with an Oscillatory Wall Temperature
}

\author{
Sankar Kumar Guchhait ${ }^{1}$, Sanatan Das ${ }^{2}$, Rabindra Nath Jana ${ }^{1}$ \\ ${ }^{1}$ Department of Applied Mathematics, Vidyasagar University, Midnapore, India \\ ${ }^{2}$ Department of Mathematics, University of Gour Banga, English Bazar, India \\ Email: jana261171@yahoo.co.in
}

Received December 11, 2012; revised January 15, 2013; accepted January 26, 2013

\begin{abstract}
The combined effects of Hall current and radiation on an unsteady MHD free convective flow of a viscous incompressible electrically conducting fluid in a vertical channel with an oscillatory wall temperature have been studied. We have considered two different cases 1) flow due to the impulsive motion of one of the channel walls and 2) flow due to the accelerated motion of one of the channel walls. The governing equations are solved analytically using the Laplace transform technique. It is found that the primary velocity and the magnitude of the secondary velocity increased with an increase in Hall parameter for the impulsive as well as the accelerated motions of one of the channel walls. An increase in either radiation parameter or frequency parameter leads to decrease in the primary velocity and the magnitude of the secondary velocity for the impulsive as well as accelerated motions of one of the channel walls. The fluid temperature decreases with an increase in radiation parameter. Further, the shear stresses at the left wall reduce with an increase in either radiation parameter or frequency parameter for the impulsive as well as the accelerated motions of one of the channel wall.
\end{abstract}

Keywords: Hall Current; MHD Free Convection; Radiation; Prandtl Number; Grashof Number; Frequency Parameter; Impulsive Motion; Accelerated Motion

\section{Introduction}

The mechanism of conduction in ionized gases in the presence of a strong magnetic field is different from that in metallic substance. The electric current in ionized gases is generally carried by electrons, which undergos successive collisions with other charged or neutral particles. In the ionized gases, the current is not proportional to the applied potential except when the field is very weak in an ionized gas where the density is low and the magnetic field is very strong, the conductivity normal to the magnetic field is reduced due to the free spiraling of electrons and ions about the magnetic lines of force before suffering collisions and a current is induced in a direction normal to both electric and magnetic fields. This phenomenon, well known in the literature, is called the Hall effect. The study of hydromagnetic flows with Hall currents has important engineering applications in problems of magnetohydrodynamic generators and of Hall accelerators as well as in flight magnetohydrodynamics. It is well known that a number of astronomical bodies posses fluid interiors and magnetic fields. It is also important in the solar physics involved in the sunspot development, the solar cycle and the structure of magnetic stars. In space technology applications and at higher operating temperatures, radiation effects can be quite significant. The radiative convective flows are frequently encountered in many scientific and environmental processes, such as astrophysical flows, water evaporation from open reservoirs, heating and cooling of chambers, and solar power technology. The unsteady hydromagnetic flow of a viscous incompressible electrically conducting fluid through a vertical channel is of considerable interest in the technical field due to its frequent occurrence in industrial and technological applications. The Hall effects on the flow of ionized gas between parallel plates under transverse magnetic field have been studied by Sato [1]. Miyatake and Fujii [2] have discussed the free convection flow between vertical plates - one plate isothermally heated and other thermally insulated. Natural convection flow between vertical parallel plates - one plate with a uniform heat flux and the other thermally insulated has been investigated by Tanaka et al. [3]. Gupta and Gupta [4] have studied the radiation effect on hydromagnetic convection in a vertical channel. Hall effects on the hydromagnetic convective 
flow through a vertical channel with conducting walls have been investigated by Dutta and Jana [5]. The unsteady hydromagnetic free convective flow with radiative heat transfer in a rotating fluid has been described by Bestman and Adjepong [6]. Joshi [7] has studied the transient effects in natural convection cooling of vertical parallel plates. Singh [8] have described the natural convection in unsteady Couette motion. Singh et al. [9] have studied the unsteady free convective flow between two vertical parallel plates. The natural convection in unsteady MHD Couette flow with heat and mass transfers has been analyzed by Jha [10]. Narahari et al. [11] have studied the unsteady free convective flow between long vertical parallel plates with constant heat flux at one boundary. The unsteady free convective flow in a vertical channel due to symmetric heating have been described by Jha et al. [12]. Singh and Paul [13] have studied the unsteady natural convective between two vertical walls heated/cooled asymmetrically. Sanyal and Adhikari [14] have studied the effects of radiation on MHD vertical channel flow. The radiation effects on MHD Couette flow with heat transfer between two parallel plates have been examined by Mebine [15]. Grosan [16] has studied the thermal radiation effect on the fully developed mixed convective flow in a vertical channel. Guria and Jana [17] have discussed Hall effects on the hydromagnetic convective flow through a rotating channel under general wall conditions. Jha and Ajibade [18] have studied the unsteady free convective Couette flow of heat generating/absorbing fluid. Effects of thermal radiation and free convection currents on the unsteady Couette flow between two vertical parallel plates with constant heat flux at one boundary have been studied by Narahari [19]. Rajput and Sahu [20] have studied the unsteady free convection MHD flow between two long vertical parallel plates with constant temperature and variable mass diffusion. Das et al. [21] have studied the radiation effects on free convection MHD Couette flow started exponentially with variable wall temperature in the presence of heat generation. The effect of radiation on transient natural convection flow between two vertical walls have been described by Mandal et al. [22]. Das et al. [23] have studied the radiation effects on unsteady MHD free convective Couette flow of heat generation/absorbing fluid. The effects of radiation on MHD free convective Couette flow in a rotating system have been discussed by Sarkar et al. [24]. Sarkar et al. [25] have studied an oscillatory MHD free convective flow between two vertical walls in a rotating system.

The aim of the present paper is to study the combined effects of Hall current and radiation on the unsteady MHD free convective flow of a viscous incompressible electrically conducting fluid in a vertical channel with an oscillatory wall temperature of one of the channel walls. It is found that the primary velocity $u_{1}$ and the magnitude of the secondary velocity $v_{1}$ decrease with an increase in either radiation parameter $R$ or frequency parameter $n$ or Prandtl number $\operatorname{Pr}$ for the impulsive as well as the accelerated motions of one of the channel walls. It is also observed that the primary velocity $u_{1}$ and the magnitude of the secondary velocity $v_{1}$ increase with an increase in either Hall parameter $m$ or Grashof number $\mathrm{Gr}$ or time $\tau$ for the impulsive as well as accelerated motions. An increase in Grashof number Gr leads to fall the fluid velocity components. An increase in the radiation parameter $R$ leads to increase the fluid temperature. Further, the shear stress $\tau_{x}$ at the wall $\eta=0$ due to the primary flow and the absolute value of the shear stress $\tau_{y}$ at the wall $\eta=0$ due to the secondary flow decrease for the impulsive as well as accelerated motions of one of the channel walls with an increase in radiation parameter $R$. The rate of heat transfer $-\theta^{\prime}(0, \tau)$ at the wall $\eta=0$ decreases while the rate of heat transfer $-\theta^{\prime}(0, \tau)$ at the wall $\eta=1$ increases with an increase in Prandtl number $\mathrm{Pr}$.

\section{Formulation of the Problem and Its Solution}

Consider the unsteady MHD flow of a viscous incompressible electrically conducting radiative fluid between two infinitely long vertical parallel walls separated by a distance $h$. The flow is set up by the buoyancy force arising from the temperature gradient. Choose a Cartesian co-ordinates system with the $x$-axis along the channel wall at $y=0$ in the vertically upward direction, the $y$-axis perpendicular to the channel walls and $z$-axis is normal to the $x y$-plane (see Figure 1). Initially, at time $t \leq 0$, the two walls and the fluid are assumed to be at the same temperature $T_{h}$ and stationary. At time $t>0$, the wall at $y=0$ starts to move in its own plane with a velocity $U(t)$ and its temperature is raised to $T_{h}+\left(T_{0}-T_{h}\right) \cos \omega t$ whereas the wall at $y=h$ is stationary and maintained at a constant temperature $T_{h}$, where $\omega$ is the frequency of the temperature oscillations. A uniform transverse magnetic field $B_{0}$ is applied perpendicular to the channel walls. We assume that the flow is laminar and the pressure gradient term in the momentum equation can be neglected. It is assumed that the effect of viscous and Joule dissipations are negligible. It is also assumed that the radiative heat flux in the $x$-direction is negligible as compared to that in the $y$-direction. As the channel walls are infinitely long, the velocity field and temperature distribution are functions of $y$ and $t$ only.

Under the usual Boussinesq approximation, the flow is governed by the following Navier-Stokes equations

$$
\frac{\partial u}{\partial t}=v \frac{\partial^{2} u}{\partial y^{2}}+g \beta\left(T-T_{h}\right)+\frac{B_{0}}{\rho} j_{y},
$$




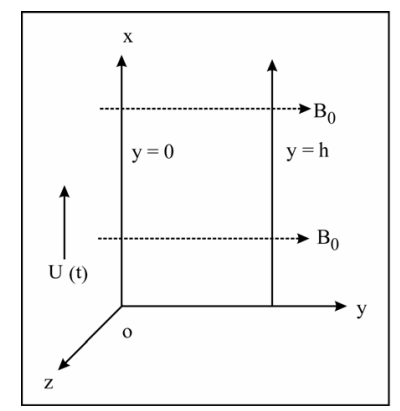

Figure 1. Geometry of the problem.

$$
\frac{\partial v}{\partial t}=v \frac{\partial^{2} v}{\partial y^{2}}-\frac{B_{0}}{\rho} j_{x}
$$

where $\rho$ is the fluid density, $v$ the kinematic viscosity, $u$ and $v$ are fluid velocity components and $g$ the acceleration due to gravity.

The energy equation is

$$
\rho c_{p} \frac{\partial T}{\partial t}=k^{*} \frac{\partial^{2} T}{\partial y^{2}}-\frac{\partial q_{r}}{\partial y},
$$

$T$ the fluid temperature, $k^{*}$ the thermal conductivity, $c_{p}$ the specific heat at constant pressure and $q_{r}$ the radiative heat flux.

The initial and boundary conditions for the velocity and temperature distributions are

$$
\begin{aligned}
& t \leq 0: u=v=0, T=T_{h} \text { for } 0 \leq y \leq h, \\
& t>0:\left\{\begin{array}{l}
u=U(t), v=0, \\
T=T_{h}+\left(T_{0}-T_{h}\right) \cos \omega t \text { at } y=0,
\end{array}\right. \\
& t>0: u=v=0, T=T_{h} \text { at } y=h .
\end{aligned}
$$

It has been shown by Cogley et al. [26] that in the optically thin limit for a non-gray gas near equilibrium, the following relation holds

$$
\frac{\partial q_{r}}{\partial y}=4\left(T-T_{h}\right) \int_{0}^{\infty} K_{\lambda_{h}}\left(\frac{\partial e_{\lambda p}}{\partial T}\right)_{h} \mathrm{~d} \lambda
$$

where $K_{\lambda_{h}}$ is the absorption coefficient, $\lambda$ is the wave length, $e_{\lambda p}$ is the Planck's function and subscript ' $h$ ' indicates that all quantities have been evaluated at the temperature $T_{h}$ which is the temperature of the walls at time $t \leq 0$. Thus, our study is limited to small difference of wall temperatures to the fluid temperature.

On the use of the Equation (5), the Equation (3) becomes

$$
\rho c_{p} \frac{\partial T}{\partial t}=k^{*} \frac{\partial^{2} T}{\partial y^{2}}-4\left(T-T_{h}\right) I
$$

where

$$
I=\int_{0}^{\infty} K_{\lambda_{h}}\left(\frac{\partial e_{\lambda p}}{\partial T}\right)_{h} \mathrm{~d} \lambda
$$

The generalized Ohm's law, on taking Hall currents into account and neglecting ion-slip and thermo-electric effect, is (see Cowling [27])

$$
\boldsymbol{j}+\frac{\omega_{e} \tau_{e}}{B_{0}}(\boldsymbol{j} \times \boldsymbol{B})=\sigma(\boldsymbol{E}+\boldsymbol{q} \times \boldsymbol{B}),
$$

where $\boldsymbol{j}$ is the current density vector, $\boldsymbol{B}$ the magnetic field vector, $\boldsymbol{E}$ the electric field vector, $\omega_{e}$ the cyclotron frequency, $\sigma$ the electrical conductivity of the fluid and $\tau_{e}$ the collision time of electron.

We shall assume that the magnetic Reynolds number for the flow is small so that the induced magnetic field can be neglected. This assumption is justified since the magnetic Reynolds number is generally very small for partially ionized gases. The solenoidal relation $\nabla \cdot \boldsymbol{B}=0$ for the magnetic field gives $B_{z}=B_{0}=$ constant everywhere in the fluid where $\boldsymbol{B} \equiv\left(0,0, B_{0}\right)$. Further, if $\left(j_{x}, j_{y}, j_{z}\right)$ be the components of the current density $\boldsymbol{j}$, then the equation of the conservation of the current density $\nabla \cdot \boldsymbol{j}=0$ gives $j_{z}=$ constant. This constant is zero since $j_{z}=0$ at the walls which are electrically non- conducting. Thus $j_{z}=0$ everywhere in the flow. Since the induced magnetic field is neglected, the

Maxwell's equation $\nabla \times \boldsymbol{E}=-\frac{\partial \boldsymbol{B}}{\partial t}$ becomes $\nabla \times \boldsymbol{E}=0$ which gives $\frac{\partial E_{x}}{\partial z}=0$ and $\frac{\partial E_{y}}{\partial z}=0$. This implies that $E_{x}=$ constant and $E_{y}=$ constant everywhere in the flow. We choose this constants equal to zero, i.e. $E_{x}=E_{y}=0$.

In view of the above assumption, the Equation (8) gives

$$
\begin{aligned}
& j_{x}+m j_{y}=\sigma v B_{0}, \\
& j_{y}-m j_{x}=-\sigma u B_{0},
\end{aligned}
$$

where $m=\omega_{e} \tau_{e}$ is the Hall parameter.

Solving (9) and (10) for $j_{x}$ and $j_{y}$, we have

$$
\begin{aligned}
& j_{x}=\frac{\sigma B_{0}}{1+m^{2}}(v+m u), \\
& j_{y}=\frac{\sigma B_{0}}{1+m^{2}}(m v-u) .
\end{aligned}
$$

On the use of (11) and (12), the momentum Equations (1) and (2) along $x$ - and $y$-directions become

$$
\begin{gathered}
\frac{\partial u}{\partial t}=v \frac{\partial^{2} u}{\partial y^{2}}+g \beta\left(T-T_{h}\right)-\frac{\sigma B_{0}^{2}}{\rho\left(1+m^{2}\right)}(u-m v), \\
\frac{\partial v}{\partial t}=v \frac{\partial^{2} v}{\partial y^{2}}-\frac{\sigma B_{0}^{2}}{\rho\left(1+m^{2}\right)}(v+m u) .
\end{gathered}
$$

Introducing non-dimensional variables 


$$
\begin{aligned}
& \left(u_{1}, v_{1}\right)=\frac{(u, v)}{U_{0}}, \eta=\frac{y}{h}, \tau=\frac{v t}{h^{2}}, \\
& \theta=\frac{T-T_{h}}{T_{0}-T_{h}}, U(t)=U_{0} f(\tau),
\end{aligned}
$$

Equations (6), (13) and (14) become

$$
\begin{gathered}
\frac{\partial u_{1}}{\partial \tau}=\frac{\partial^{2} u_{1}}{\partial \eta^{2}}+\operatorname{Gr} \theta-\frac{M^{2}}{1+m^{2}}\left(u_{1}-m v_{1}\right), \\
\frac{\partial v_{1}}{\partial \tau}=\frac{\partial^{2} v_{1}}{\partial \eta^{2}}-\frac{M^{2}}{1+m^{2}}\left(v_{1}+m u_{1}\right), \\
\operatorname{Pr} \frac{\partial \theta}{\partial \tau}=\frac{\partial^{2} \theta}{\partial \eta^{2}}-R \theta
\end{gathered}
$$

where $M^{2}=\sigma B_{0}^{2} h^{2} /(\rho v)$ is the magnetic parameter, $\mathrm{Gr}=g \beta\left(T_{0}-T_{h}\right) h^{2} / v^{2}$ the Grashof number,

$\operatorname{Pr}=\rho c_{p} v / k^{*}$ the Prandtl number and $R=4 I h^{2} / k^{*}$ the radiation parameter.

The initial and boundary conditions (4) become

$$
\begin{aligned}
& \tau \leq 0: u_{1}=v_{1}=0, \theta=0 \text { for } 0 \leq \eta \leq 1, \\
& \tau>0: u_{1}=f(\tau), v_{1}=0, \theta=\cos n \tau \text { at } \eta=0, \\
& \tau>0: u_{1}=v_{1}=0, \theta=0 \text { at } \eta=1,
\end{aligned}
$$

where $n=\frac{\omega h^{2}}{v}$ is the frequency parameter.

Combining Equations (16) and (17), we get

$$
\frac{\partial F}{\partial \tau}=\frac{\partial^{2} F}{\partial \eta^{2}}+\operatorname{Gr} \theta-\frac{(1+\mathrm{i} m) M^{2}}{1+m^{2}} F,
$$

where

$$
F=u_{1}+\mathrm{i} v_{1} \text { and } \mathrm{i}=\sqrt{-1} .
$$

The initial and boundary conditions for $F(\eta, \tau)$ are

$$
\begin{aligned}
& \tau \leq 0: F=0 \text { for } 0 \leq \eta \leq 1, \\
& \tau>0: F=f(\tau) \text { at } \eta=0, \\
& \tau>0: F=0 \text { at } \eta=1 .
\end{aligned}
$$

Taking the Laplace transform of Equations (20) and (18) and on the use of (19) and (22), we have

$$
\begin{aligned}
& \frac{\mathrm{d}^{2} \bar{F}}{\mathrm{~d} \eta^{2}}-(a+s) \bar{F}=-\mathrm{Gr} \bar{\theta}, \\
& \theta(\eta, \tau)= \begin{cases}\frac{1}{2}\left[\mathrm{e}^{\mathrm{i} n \tau} \frac{\sinh \sqrt{R+\mathrm{in} \operatorname{Pr}}(1-\eta)}{\sinh \sqrt{R+\mathrm{i} n \operatorname{Pr}}}+\mathrm{e}^{-\mathrm{i} n \tau} \frac{\sinh \sqrt{R-\mathrm{in} \operatorname{Pr}}(1-\eta)}{\sinh \sqrt{R-\mathrm{in} \operatorname{Pr}}}\right]+\frac{2 \pi}{\operatorname{Pr}} \sum_{k=1}^{\infty} \frac{k s_{1} \mathrm{e}^{\mathrm{s}_{1} \tau}}{s_{1}^{2}+n^{2}} \sin k \pi \eta \text { for } \operatorname{Pr} \neq 1 \\
\frac{1}{2}\left[\mathrm{e}^{\mathrm{i} n \tau} \frac{\sinh \sqrt{R+\mathrm{in}}(1-\eta)}{\sinh \sqrt{R+\mathrm{in}}}+\mathrm{e}^{-\mathrm{in} \tau} \frac{\sinh \sqrt{R-\mathrm{i} n}(1-\eta)}{\sinh \sqrt{R-\mathrm{i} n}}\right]+2 \pi \sum_{k=1}^{\infty} \frac{k s_{3} \mathrm{e}^{s_{3} \tau}}{s_{3}^{2}+n^{2}} \sin k \pi \eta \quad \text { for } \operatorname{Pr}=1,\end{cases}
\end{aligned}
$$

$$
\frac{\mathrm{d}^{2} \bar{\theta}}{\mathrm{d} \eta^{2}}-(R+s \operatorname{Pr}) \bar{\theta}=0
$$

where

$$
a=\frac{M^{2}(1+\mathrm{i} m)}{1+m^{2}} .
$$

The initial and boundary conditions for $\bar{F}(\eta, s)$ and $\bar{\theta}(\eta, s)$ are

$$
\begin{aligned}
& \bar{F}(0, s)=\bar{f}(s), \bar{F}(1, s)=0, \\
& \bar{\theta}(0, s)=\frac{1}{2}\left(\frac{1}{s-\mathrm{in}}+\frac{1}{s+\mathrm{in}}\right), \bar{\theta}(1, s)=0 .
\end{aligned}
$$

Solutions of Equations (23) and (24) subject to the boundary conditions (26) are given by

$$
\bar{\theta}(\eta, s)= \begin{cases}\frac{s}{s^{2}+n^{2}} \frac{\sinh \sqrt{R+s \operatorname{Pr}}(1-\eta)}{\sinh \sqrt{R+s \operatorname{Pr}}}, & \text { for } \operatorname{Pr} \neq 1 \\ \frac{s}{s^{2}+n^{2}} \frac{\sinh \sqrt{R+s}(1-\eta)}{\sinh \sqrt{R+s}}, & \text { for } \operatorname{Pr}=1\end{cases}
$$

$$
\begin{aligned}
& \bar{F}(\eta, s) \\
& =\left\{\begin{array}{l}
\bar{f}(s) \frac{\sinh \sqrt{a+s}(1-\eta)}{\sinh \sqrt{a+s}}+\frac{s \mathrm{Gr}}{(\operatorname{Pr}-1)(s+\alpha)\left(s^{2}+n^{2}\right)} \\
\times\left\{\frac{\sinh \sqrt{a+s}(1-\eta)}{\sinh \sqrt{a+s}}-\frac{\sinh \sqrt{R+s \operatorname{Pr}}(1-\eta)}{\sinh \sqrt{R+s \operatorname{Pr}}}\right\} \\
\bar{f}(s) \frac{\sinh \sqrt{a+s}(1-\eta)}{\sinh \sqrt{a+s}}+\frac{s \mathrm{Gr}}{(R-a)\left(s^{2}+n^{2}\right)} \\
\times\left\{\frac{\sinh \sqrt{a+s}(1-\eta)}{\sinh \sqrt{a+s}}-\frac{\sinh \sqrt{R+s}(1-\eta)}{\sinh \sqrt{R+s}}\right\} \\
\text { for } \operatorname{Pr}=1,
\end{array}\right.
\end{aligned}
$$

Now, we shall considered the following cases.

\section{1) When the wall at $\boldsymbol{\eta}=\mathbf{0}$ started impulsively:}

In this case $f(\tau)=1$, i.e. $f(s)=\frac{1}{s}$. Then the inverse Laplace transforms of Equations (27) and (28) give the 


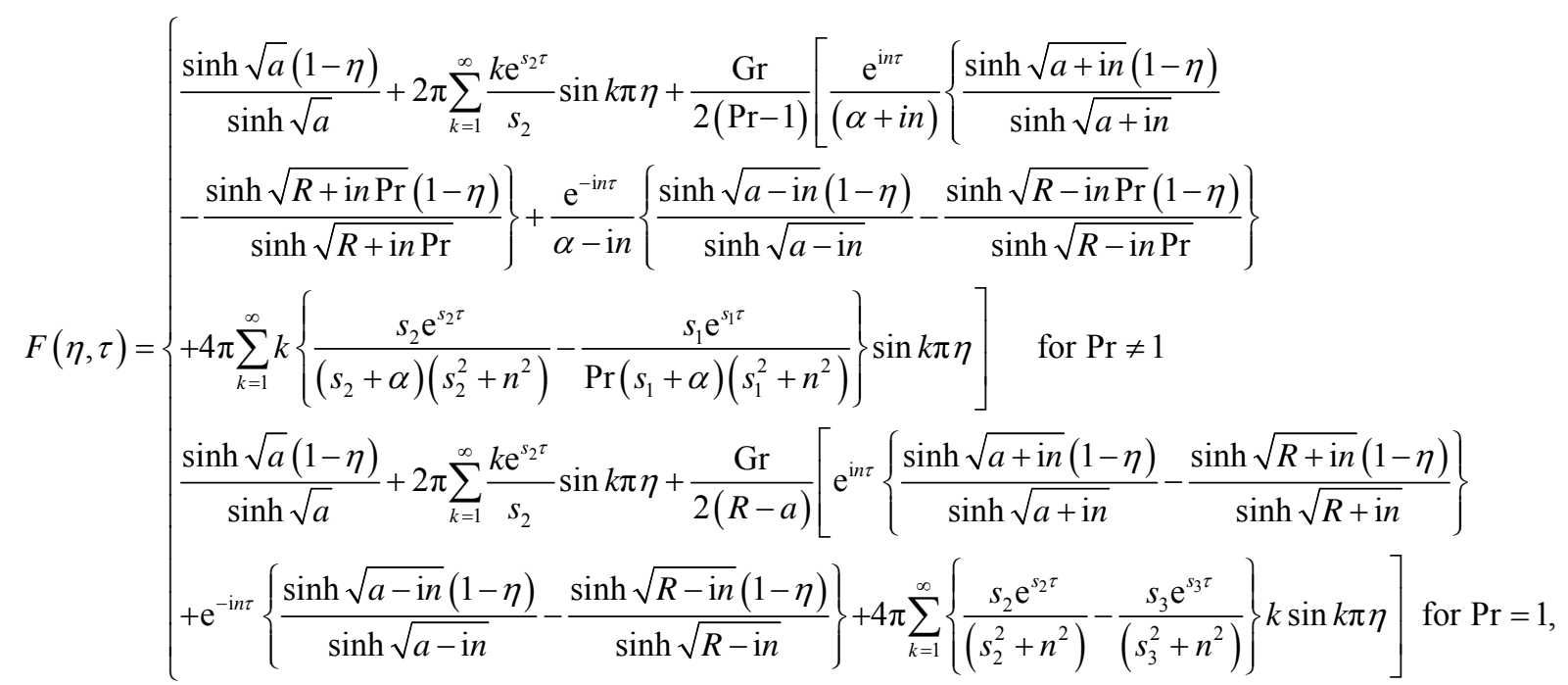

where

$$
s_{1}=-\frac{1}{\operatorname{Pr}}\left(R+k^{2} \pi^{2}\right), s_{2}=-\left[\frac{M^{2}(1+\mathrm{im})}{1+m^{2}}+k^{2} \pi^{2}\right] \text { and } s_{3}=-\left(R+k^{2} \pi^{2}\right) .
$$

\section{2) When the wall at $\eta=0$ started acceleratedly:}

In this case $f(\tau)=\tau$, i.e. $f(s)=1 / s^{2}$. Then the inverse Laplace transforms of Equations (27) and (28) give the solution for the temperature distribution and the velocity field as

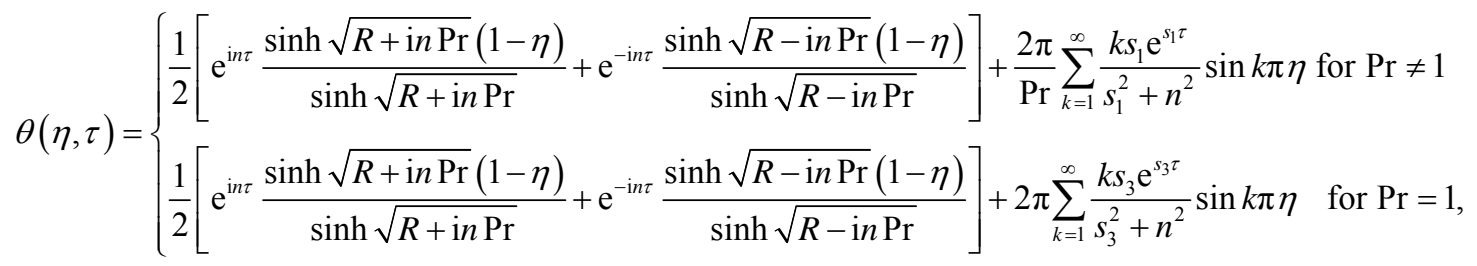

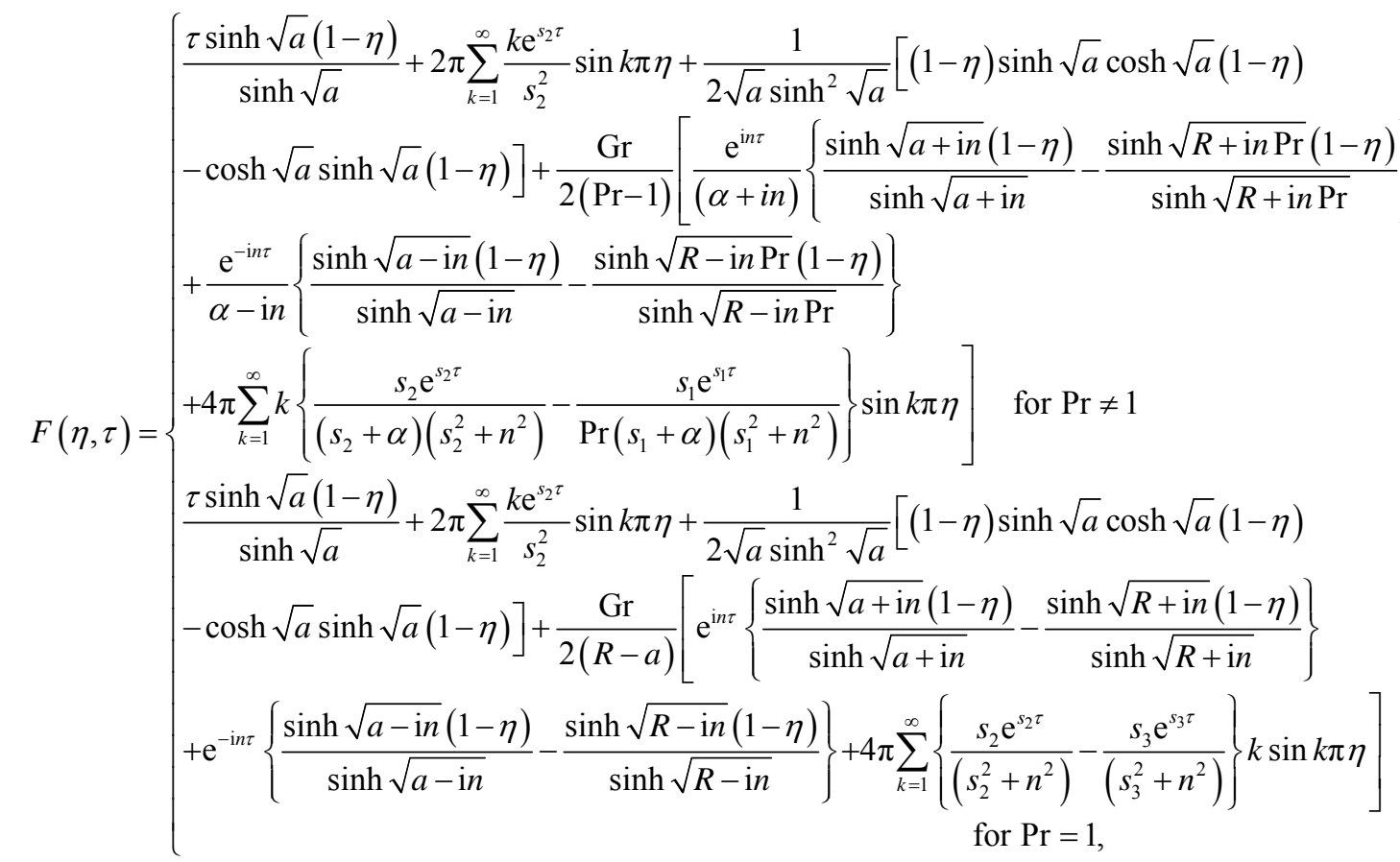


where $s_{1}, s_{2}$ and $s_{3}$ are given by (31).

\section{Results and Discussion}

We have presented the non-dimensional velocity components and temperature distribution for several values of Hall parameter $m$, radiation parameter $R$, Prandtl number $\operatorname{Pr}$, frequency parameter $n$, Grashof number Gr and time $\tau$ against $\eta$ when $M^{2}=5$ and $n \tau=\frac{\pi}{4}$ in Figures 2-17. It is seen from Figures 2 and 3 that the primary velocity $u_{1}$ and the magnitude of the secondary velocity $v_{1}$ increase with an increase of Hall parameter $m$ for the impulsive as well as accelerated motions of one of the channel walls. Figures 4 and 5 show that the primary velocity $u_{1}$ and the magnitude of the secondary velocity $v_{1}$ decrease with an increase in radiation parameter $R$ for both the impulsive and accelerated motions of one of the channel

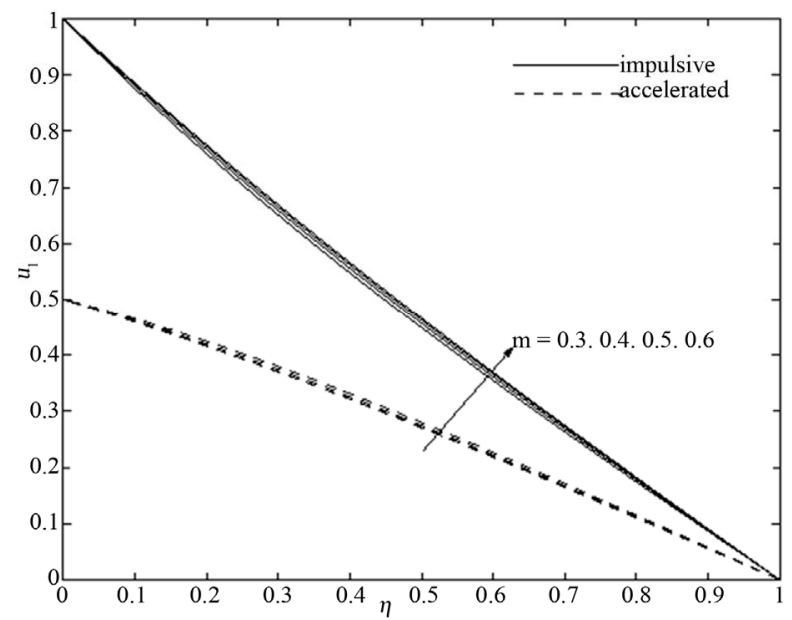

Figure 2. Primary velocity $u_{1}$ for different $m$ when $R=2, \operatorname{Pr}=0.71, n=2, \mathrm{Gr}=5$ and $\tau=0.5$.

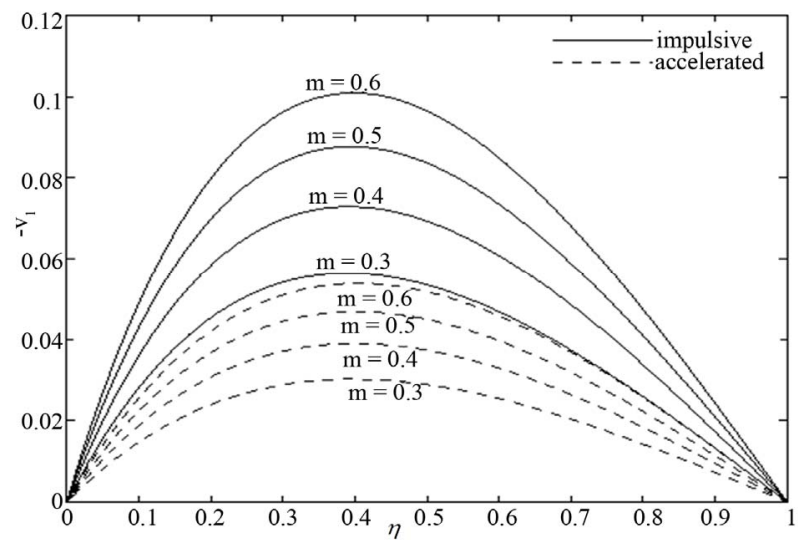

Figure 3. Secondary velocity $v_{1}$ for different $m$ when $R=2, \operatorname{Pr}=0.71, n=2, \mathrm{Gr}=5$ and $\tau=0.5$.

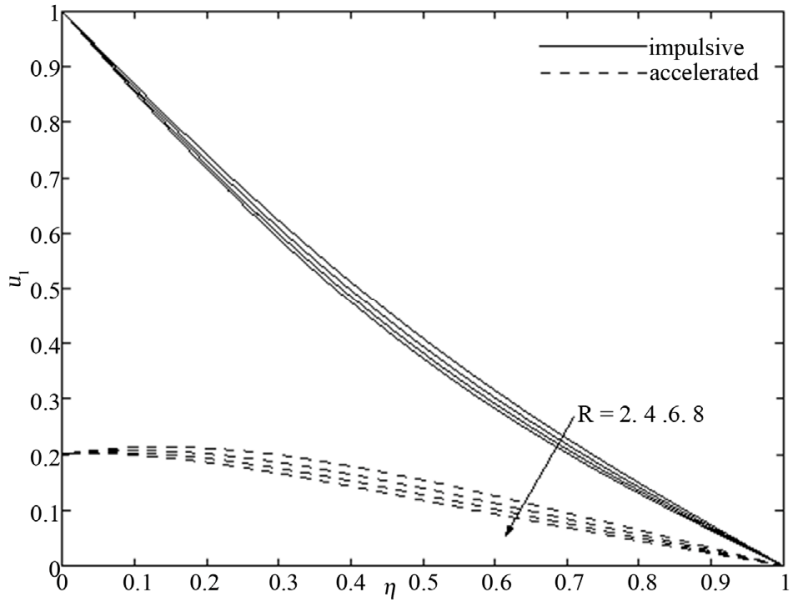

Figure 4. Primary velocity $u_{1}$ for different $R$ when $m=0.5, \operatorname{Pr}=0.71, n=2, \mathrm{Gr}=5$ and $\tau=0.2$.

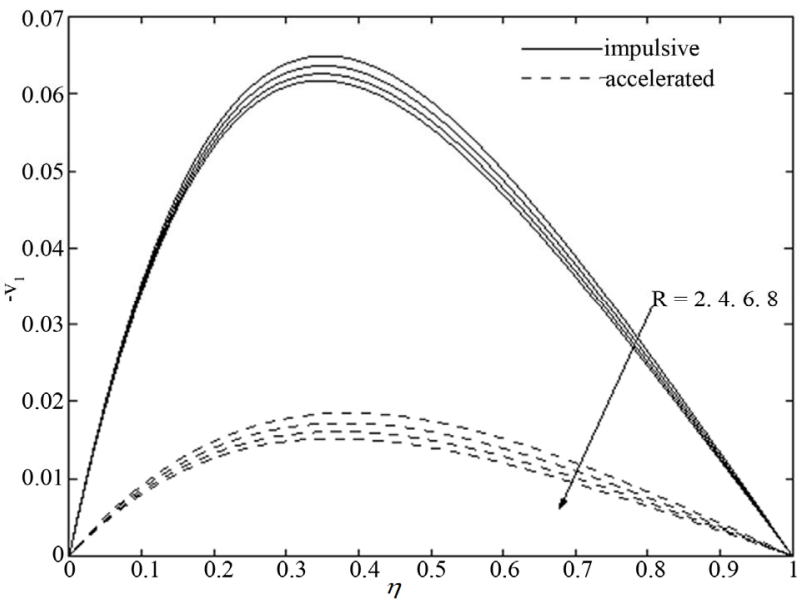

Figure 5. Secondary velocity $v_{1}$ for different $R$ when $m=0.5, \operatorname{Pr}=0.71, n=2, \mathrm{Gr}=5$ and $\tau=0.2$.

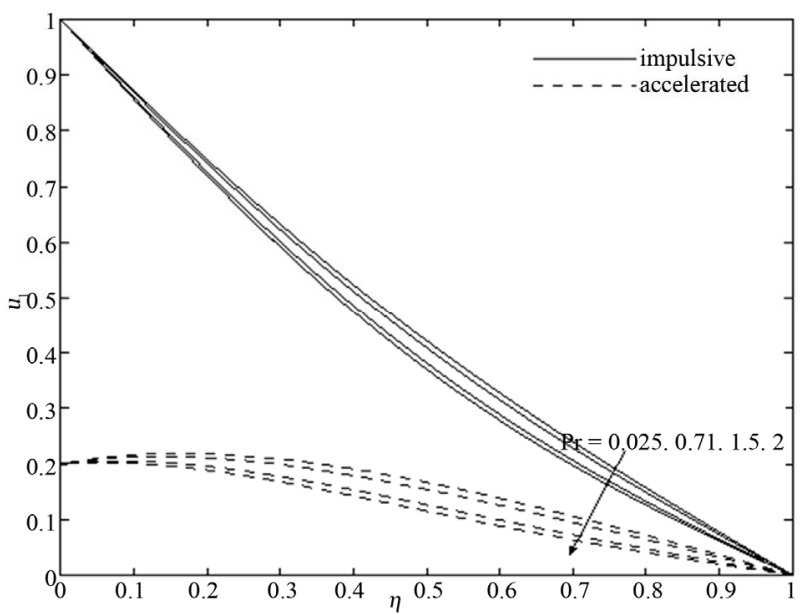

Figure 6. Primary velocity $u_{1}$ for different $\operatorname{Pr}$ when $m=0.5, R=2, n=2, \mathrm{Gr}=5$ and $\tau=0.2$. 


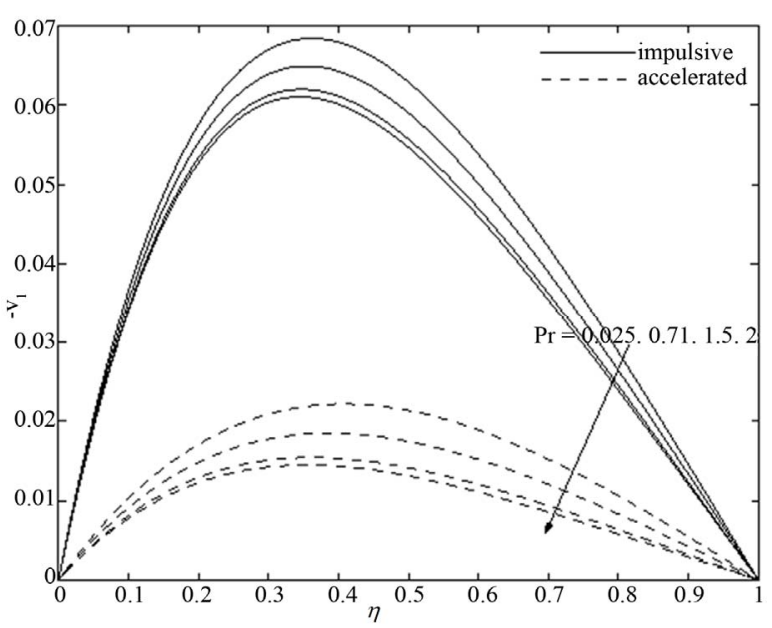

Figure 7. Secondary velocity $v_{1}$ for different $\operatorname{Pr}$ when $m=0.5, R=2, n=2, G r=5$ and $\tau=0.2$.

walls. It is illustrated from Figures 6 and 7 that the primary velocity $u_{1}$ and the magnitude of the secondary velocity $v_{1}$ decrease with an increase in Prandtl number $\operatorname{Pr}$ for the impulsive as well as accelerated motions of one of the channel walls. Figures 8 and 9 show that both the primary velocity $u_{1}$ and the magnitude of the secondary velocity $v_{1}$ decrease with an increase in frequency parameter $n$ for both the impulsive and accelerated motions of one of the channel walls. An increase in Grashof number $\mathrm{Gr}$ leads to increase the primary velocity $u_{1}$ and the magnitude of the secondary velocity $v_{1}$ for both the impulsive and accelerated motions of one of the channel walls shown in Figures 10 and 11. It is seen form Figures 12 and $\mathbf{1 3}$ that the primary velocity $u_{1}$ and the magnitude of the secondary velocity $v_{1}$ increase with an increase in time $\tau$ for both the impulsive and accelerated motions of one of the channel walls. It is seen from Figure 14 that the fluid temperature $\theta$ decreases with an increase in radiation parameter $R$. This result qualitatively agrees with expectations, since the effect of radiation is to decrease the rate of energy transport to the fluid, thereby decreasing the temperature of the fluid. It is observed from Figure 15 that the fluid temperature $\theta$ increases with an increase in Prandtl number $\mathrm{Pr}$. This is in agreement with the physical fact that the thermal boundary layer thickness decreases with increasing $\mathrm{Pr}$. Figure 16 shows that the fluid temperature $\theta$ decreases with an increase of frequency parameter $n$. Figure 17 shows that the fluid temperature $\theta$ increases when time $\tau$ progresses. It is seen from Figures 2-13 that the fluid velocities for the impulsive motion of one of the channel walls is always greater than the accelerated motion.

The rate of heat transfer at the channel walls $\eta=0$ and $\eta=1$ are respectively $\theta^{\prime}(0, \tau)\left[=\left(\frac{\partial \theta}{\partial \eta}\right)_{\eta=0}\right]$ and $\theta^{\prime}(1, \tau)\left[=\left(\frac{\partial \theta}{\partial \eta}\right)_{\eta=1}\right]$ and are given by (see the Equations (34) and (35) below).

where $s_{1}, s_{2}$ and $s_{3}$ are given by (31).

Numerical results of the rate of heat transfer at the channel walls $\eta=0$ and $\eta=1$ are respectively $-\theta^{\prime}(0, \tau)$ and $-\theta^{\prime}(1, \tau)$ which are presented in Tables 1-3 for several values of Prandtl number $\operatorname{Pr}$, time $\tau$, frequency parameter $n$ when $n \tau=\frac{\pi}{4}$. Table 1 shows that the rate of heat transfer $-\theta^{\prime}(0, \tau)$ decreases while the rate of heat transfer $-\theta^{\prime}(1, \tau)$ increases with an increase in Prandtl number $\operatorname{Pr}$. Table 2 shows that the rate of heat transfer $-\theta^{\prime}(0, \tau)$ at the wall $\eta=0$ decreases whereas the rate of heat transfer $-\theta^{\prime}(1, \tau)$ at the wall $\eta=1$ increases when time $\tau$ progresses. It is seen from Table 3 that the rate of heat transfers $-\theta^{\prime}(0, \tau)$ and $-\theta^{\prime}(1, \tau)$ decrease with an increase in frequency parameter $n$.

For the impulsive motion, the non-dimensional shear stress at the wall $\eta=0$ is given by (see the Equations (36) and (37) below).

$$
\begin{aligned}
& -\theta^{\prime}(0, \tau)=\left\{\begin{array}{l}
\frac{1}{2}\left[\left[\mathrm{e}^{\mathrm{i} i} \sqrt{R+\mathrm{in} \operatorname{Pr}} \operatorname{coth} \sqrt{R+\mathrm{i} n \operatorname{Pr}}+\mathrm{e}^{-\mathrm{i} n \tau} \sqrt{R-\mathrm{in} \operatorname{Pr}} \operatorname{coth} \sqrt{R-\mathrm{in} \operatorname{Pr}}\right]-\frac{2 \pi^{2}}{\operatorname{Pr}} \sum_{k=1}^{\infty} \frac{k^{2} s_{1} \mathrm{e}^{s_{1} \tau}}{s_{1}^{2}+n^{2}} \text { for Pr } \neq 1\right. \\
\frac{1}{2}\left[\mathrm{e}^{\mathrm{i} n \tau} \sqrt{R+\mathrm{in} \operatorname{Pr}} \operatorname{coth} \sqrt{R+\mathrm{i} n}+\mathrm{e}^{-\mathrm{i} n \tau} \sqrt{R-\mathrm{i} n} \operatorname{coth} \sqrt{R-\mathrm{i} n}\right]-2 \pi^{2} \sum_{k=1}^{\infty} \frac{k^{2} s_{3} \mathrm{e}^{s_{3} \tau}}{s_{3}^{2}+n^{2}} \quad \text { for } \operatorname{Pr}=1
\end{array}\right.
\end{aligned}
$$

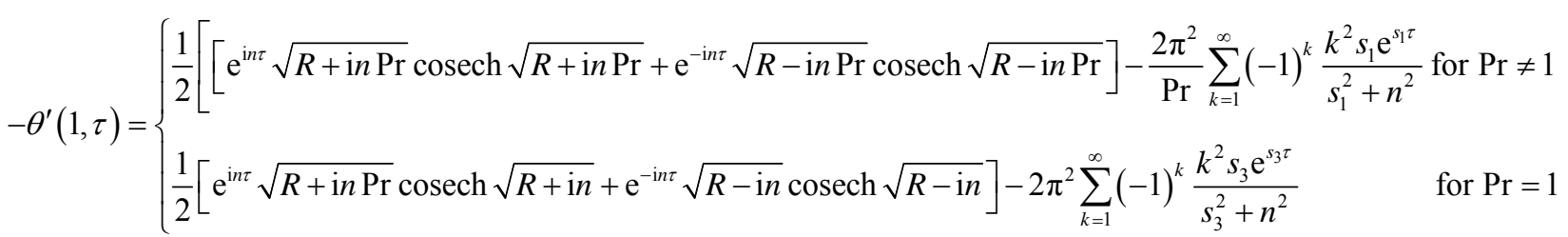




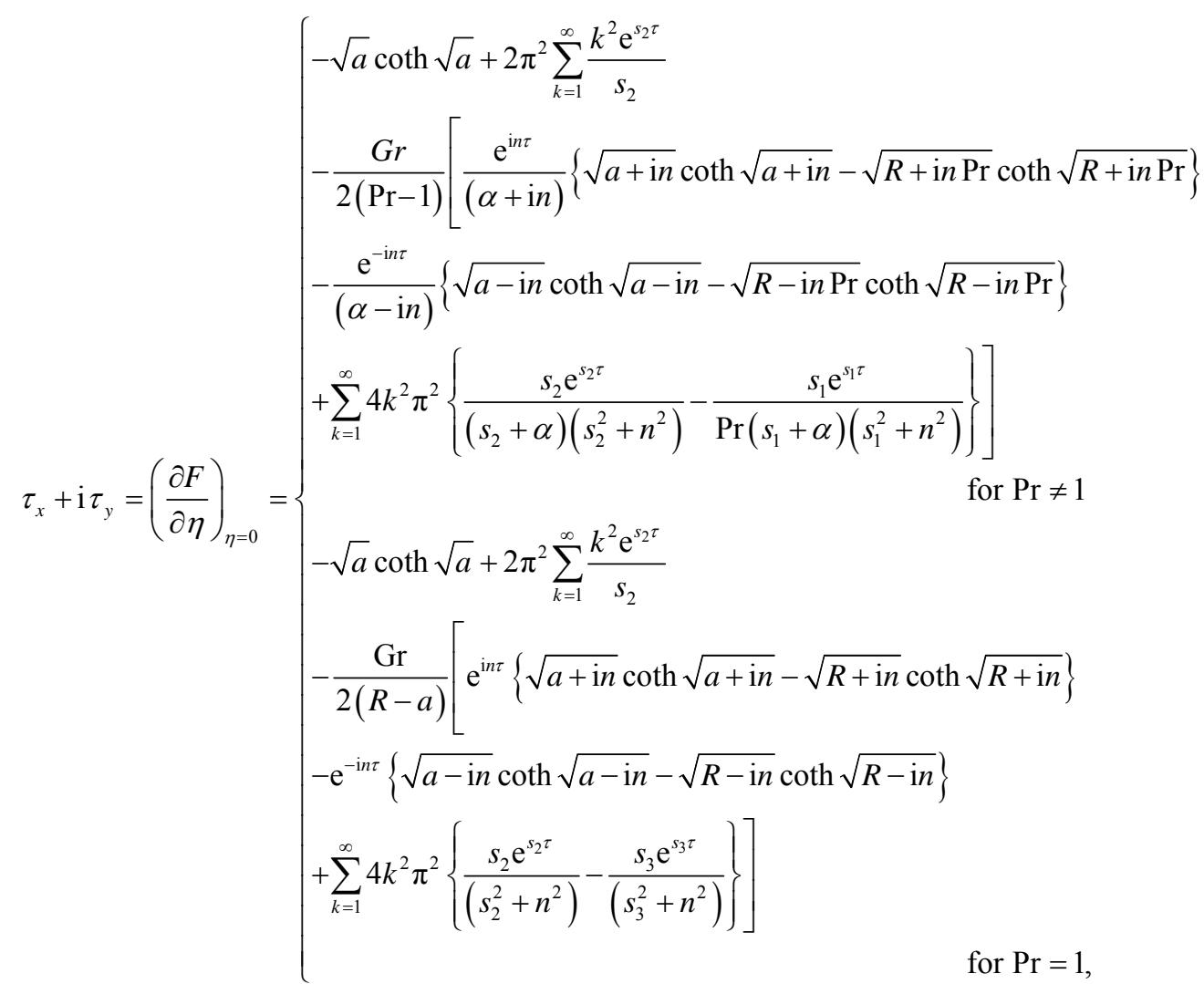

where $s_{1}, s_{2}$ and $s_{3}$ are given by (31).

For the accelerated motion, the non-dimensional shear stress at the wall $\eta=0$ is as

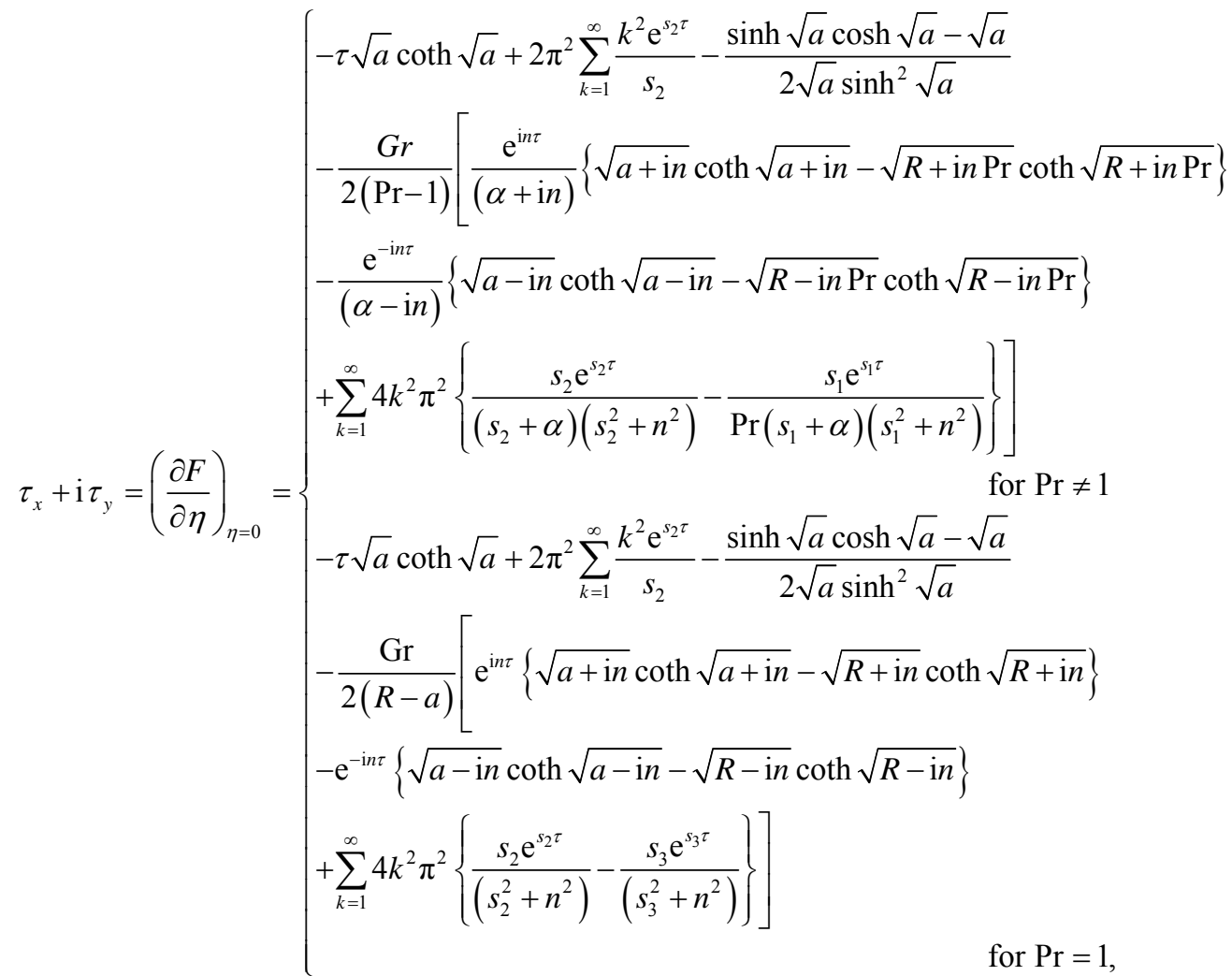

where $s_{1}, s_{2}$ and $s_{3}$ are given by (31). 


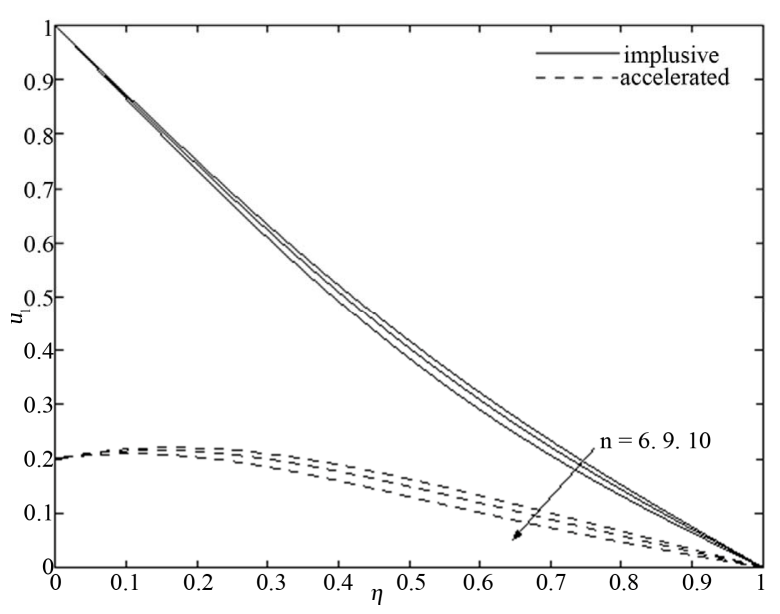

Figure 8. Primary velocity $u_{1}$ for different $n$ when $m=0.5, R=2, \operatorname{Pr}=0.71, \mathrm{Gr}=5$ and $\tau=0.2$.

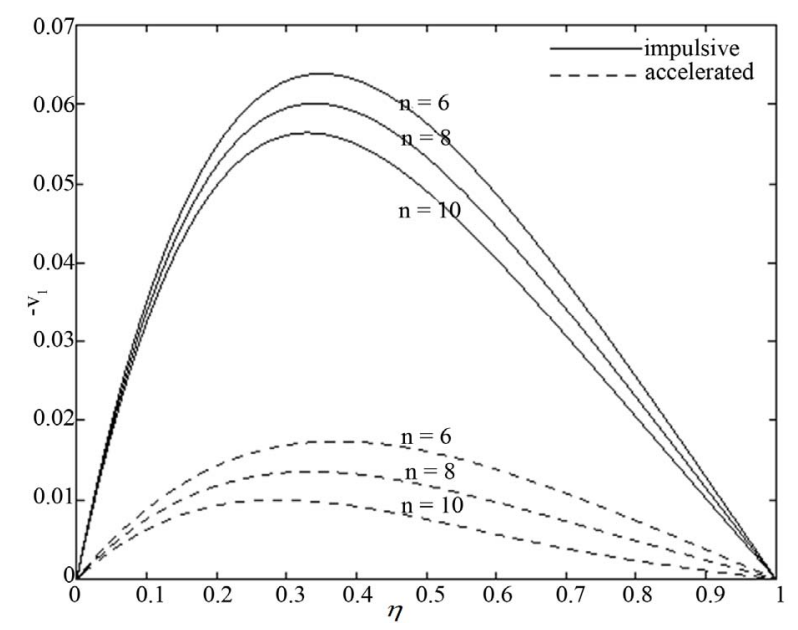

Figure 9. Secondary velocity $v_{1}$ for different $n$ when $m=0.5, R=2, \operatorname{Pr}=0.71, \mathrm{Gr}=5$ and $\tau=0.2$.

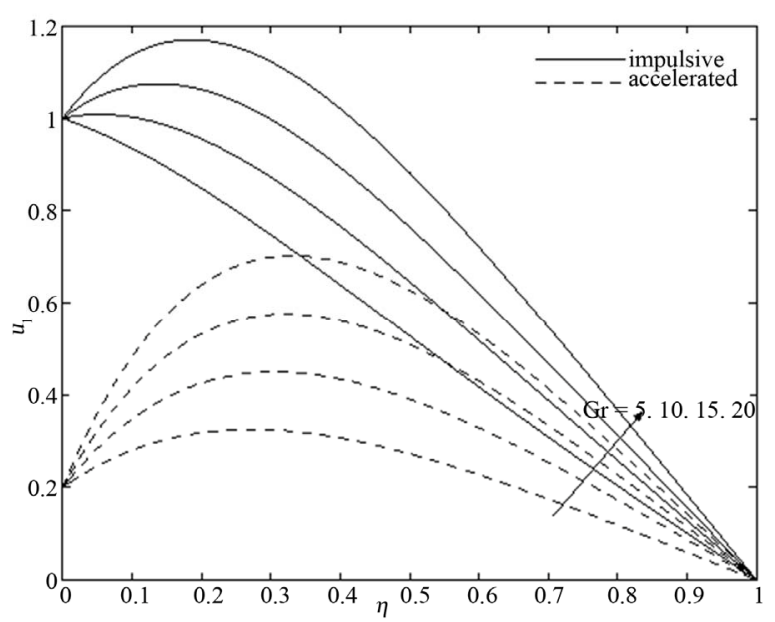

Figure 10. Primary velocity $u_{1}$ for different $\mathrm{Gr}$ when $m=0.5, R=2, \operatorname{Pr}=0.71, n=2$ and $\tau=0.2$.

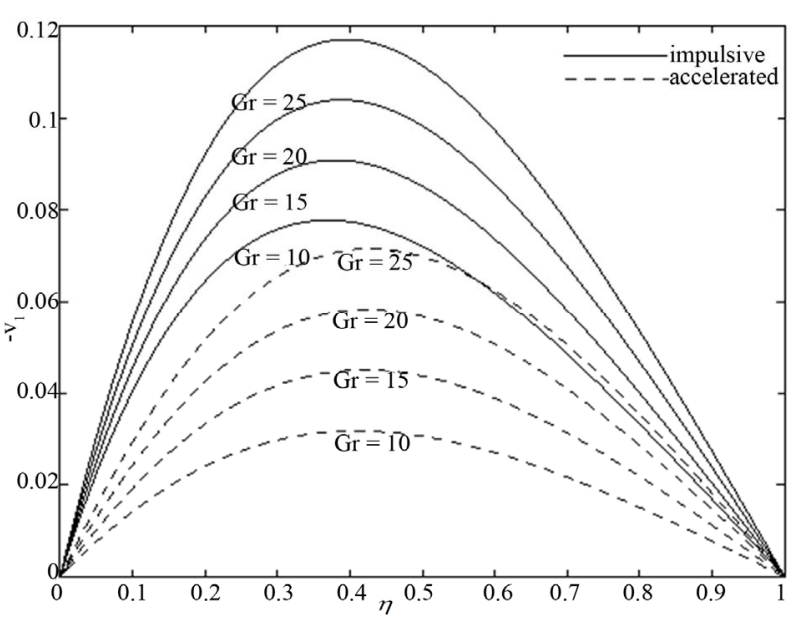

Figure 11. Secondary velocity $v_{1}$ for different $\mathrm{Gr}$ when $m=0.5, R=2, \operatorname{Pr}=0.71, n=2$ and $\tau=0.2$.

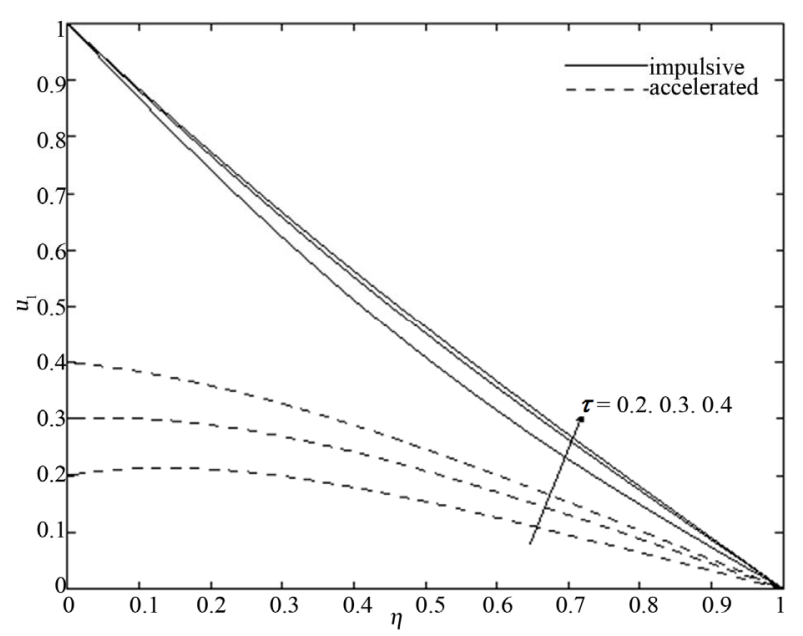

Figure 12. Primary velocity $u_{1}$ for different $\tau$ when $m=0.5, R=2, \operatorname{Pr}=0.71, n=2$ and $M^{2}=5$.

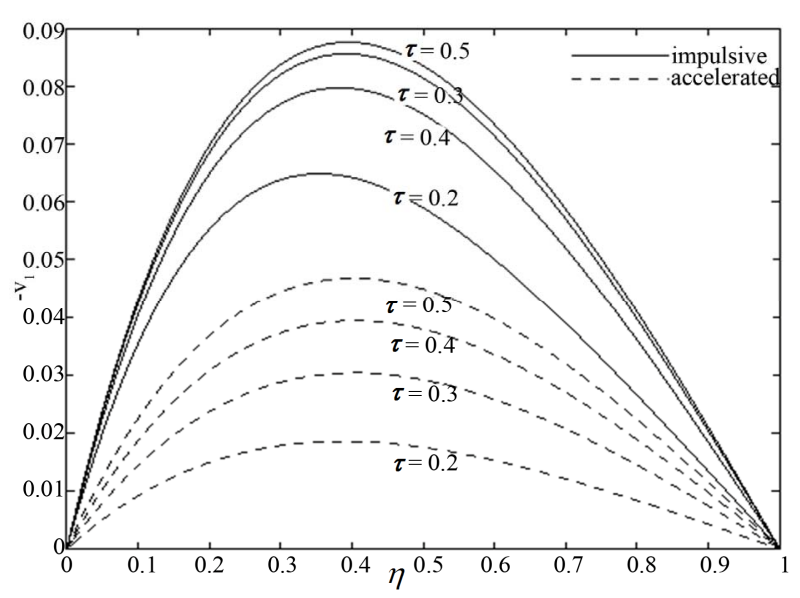

Figure 13. Secondary velocity $v_{1}$ for different $\tau$ when $m=0.5, R=2, \operatorname{Pr}=0.71, n=2$ and $M^{2}=5$. 
Table 1. Rate of heat transfer $-\theta^{\prime}(0, \tau)$ and $-\theta^{\prime}(1, \tau)$ when $n=2$ and $\tau=0.5$.

\begin{tabular}{cccccccccc}
\hline \multicolumn{9}{c}{$-\theta^{\prime}(0, \tau)$} & \multicolumn{3}{c}{$-\theta^{\prime}(1, \tau)$} \\
\hline$R \backslash \operatorname{Pr}$ & 0.025 & 0.50 & 0.71 & 1.5 & 0.025 & 0.50 & 0.71 & 1.5 \\
0.5 & 0.81019 & 0.61501 & 0.53916 & 0.35855 & 0.65667 & 0.74367 & 0.77283 & 0.78451 \\
1.0 & 0.91808 & 0.73317 & 0.66030 & 0.47547 & 0.60637 & 0.68479 & 0.71169 & 0.73004 \\
1.5 & 1.01988 & 0.84411 & 0.77404 & 0.58682 & 0.56124 & 0.63216 & 0.65695 & 0.68005 \\
2.0 & 1.11631 & 0.94872 & 0.88126 & 0.69306 & 0.52058 & 0.58492 & 0.60777 & 0.63414 \\
\hline
\end{tabular}

Table 2. Rate of heat transfer $-\theta^{\prime}(0, \tau)$ and $-\theta^{\prime}(1, \tau)$ when $n=2$ and $\operatorname{Pr}=2$.

\begin{tabular}{ccccccccc}
\hline \multicolumn{9}{c}{$-\theta^{\prime}(0, \tau)$} \\
\hline$R \backslash \tau$ & 0.1 & 0.2 & 0.3 & 0.4 & 0.1 & 0.2 & 0.3 & 0.4 \\
\hline 0.5 & 1.47365 & 0.82218 & 0.55322 & 0.40750 & 0.09949 & 0.29145 & 0.49835 & 0.63567 \\
1.0 & 1.53123 & 0.89769 & 0.64202 & 0.50682 & 0.08631 & 0.27539 & 0.47252 & 0.59998 \\
1.5 & 1.58644 & 0.97084 & 0.72809 & 0.60282 & 0.07517 & 0.26086 & 0.44838 & 0.56653 \\
2.0 & 1.63963 & 1.04186 & 0.81162 & 0.69566 & 0.06571 & 0.24763 & 0.42577 & 0.53517 \\
\hline
\end{tabular}

Table 3. Rate of heat transfer $-\theta^{\prime}(0, \tau)$ and $-\theta^{\prime}(1, \tau)$ when $\tau=0.5$ and $\operatorname{Pr}=2$.

\begin{tabular}{cccccccccc}
\hline \multicolumn{9}{c}{$-\theta^{\prime}(0, \tau)$} & \multicolumn{3}{c}{$-\theta^{\prime}(1, \tau)$} \\
\hline$R \backslash n$ & 2 & 3 & 4 & 5 & 2 & 3 & 4 & 5 \\
0.5 & 0.32266 & 0.18085 & 0.11582 & 0.09219 & 0.71936 & 0.67272 & 0.56856 & 0.44258 \\
1.0 & 0.43004 & 0.28113 & 0.20490 & 0.17048 & 0.67574 & 0.63838 & 0.54692 & 0.43254 \\
1.5 & 0.53342 & 0.37888 & 0.29290 & 0.24863 & 0.63501 & 0.60540 & 0.52516 & 0.42146 \\
2.0 & 0.63301 & 0.47408 & 0.37966 & 0.32643 & 0.59701 & 0.57386 & 0.50352 & 0.40961 \\
\hline
\end{tabular}

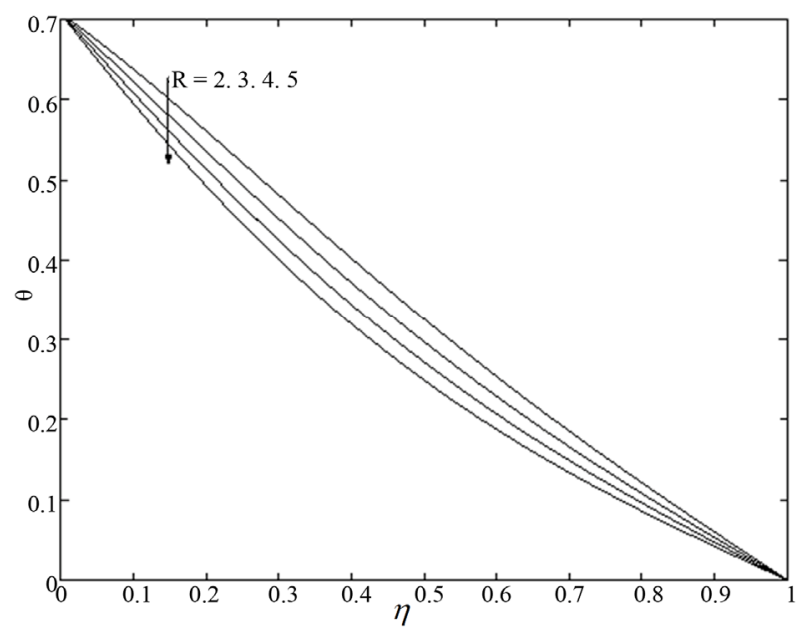

Figure 14. Temperature $\theta$ for different $R$ when $\operatorname{Pr}=0.71, n=2$ and $\tau=0.2$.

Numerical results of the non-dimensional shear stresses $\tau_{x}$ and $\tau_{y}$ at the wall $(\eta=0)$ due to the primary and the secondary flows are plotted in Figures 18-27 against Hall parameter $m$ for several values of radiation parameter $R$, Prandtl number $\operatorname{Pr}$, frequency parameter $n$, Grashof number $\mathrm{Gr}$ and time $\tau$ when

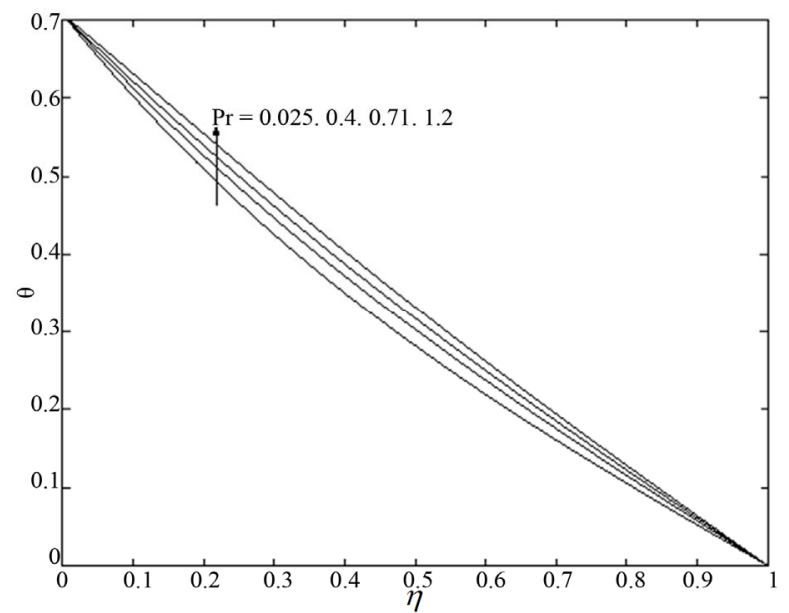

Figure 15. Temperature $\theta$ for different $\operatorname{Pr}$ when $R=2, n=2$ and $\tau=0.2$.

$M^{2}=5$ and $n \tau=\frac{\pi}{4}$. Figures 18 and 19 show that the shear stress $\tau_{x}$ due to the primary flow and the magnitude of the shear stress $\tau_{y}$ due to the secondary flow at the wall $\eta=0$ decrease for the impulsive as well as accelerated motions of one of the channel 


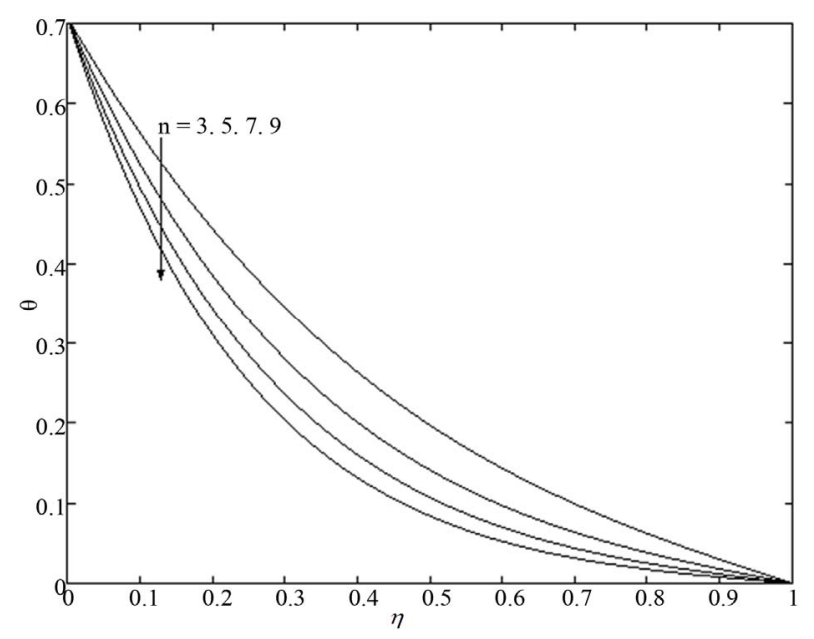

Figure 16. Temperature $\theta$ for different $n$ for $\operatorname{Pr}=0.71, R=2$ and $\tau=0.2$.

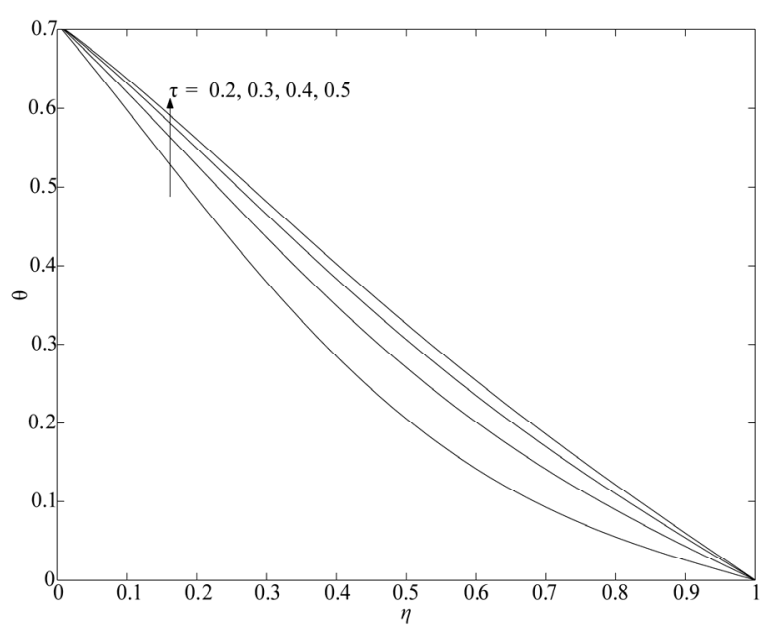

Figure 17. Temperature $\theta$ for different time $\tau$ for $\operatorname{Pr}=0.71, n=2$ and $R=2$.

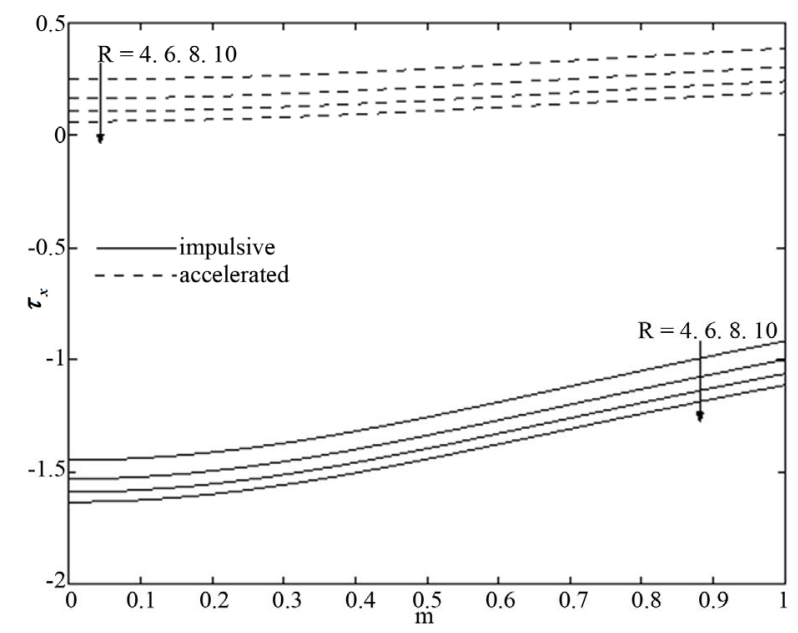

Figure 18. Shear stress $\tau_{x}$ for different $R$ when $\mathrm{Gr}=5, \operatorname{Pr}=0.71, n=2$ and $\tau=0.2$.

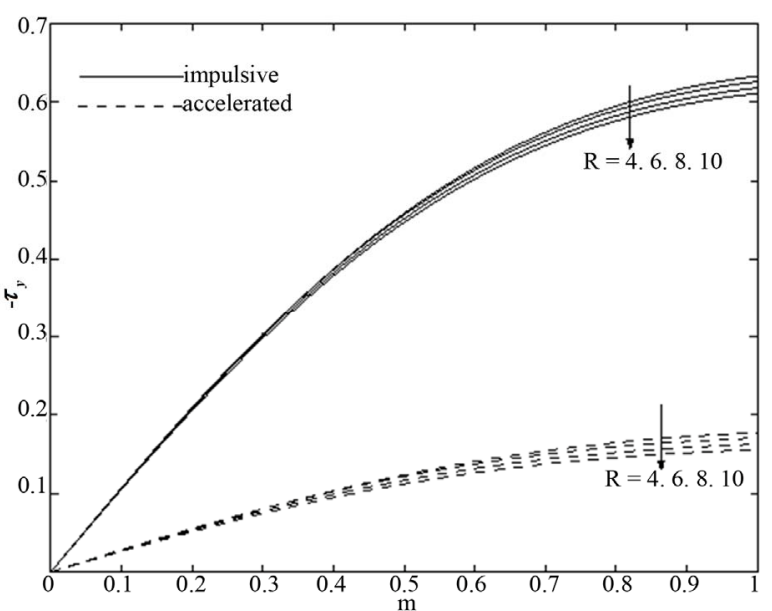

Figure 19. Shear stress $\tau_{y}$ for different $R$ when $\operatorname{Pr}=0.71, n=2, \mathrm{Gr}=5$ and $\tau=0.2$.

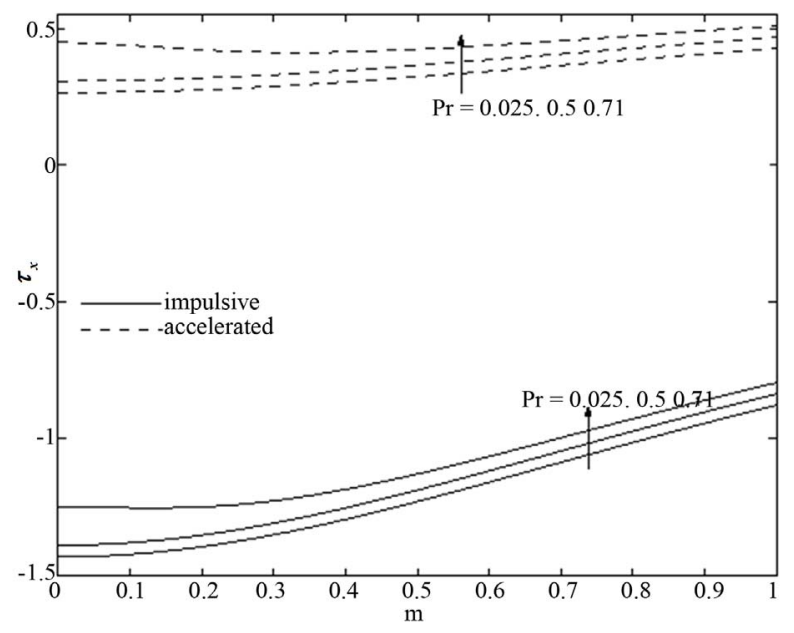

Figure 20. Shear stress $\tau_{x}$ for different Pr when $\mathrm{R}=2, \mathrm{n}=2, \mathrm{Gr}=5$ and $\tau=0.2$.

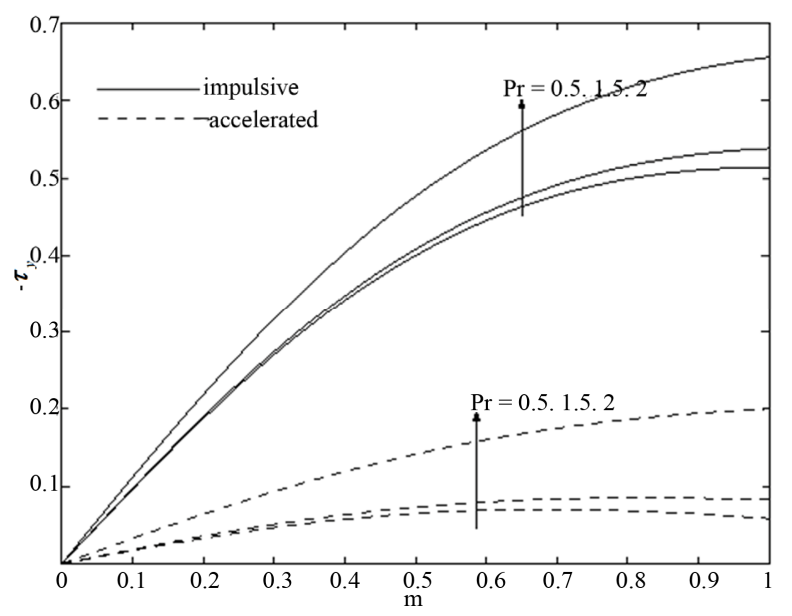

Figure 21. Shear stress $\tau_{y}$ for different $\operatorname{Pr}$ when $R=2, n=2, \tau=0.2$ and $\mathrm{Gr}=5$. 


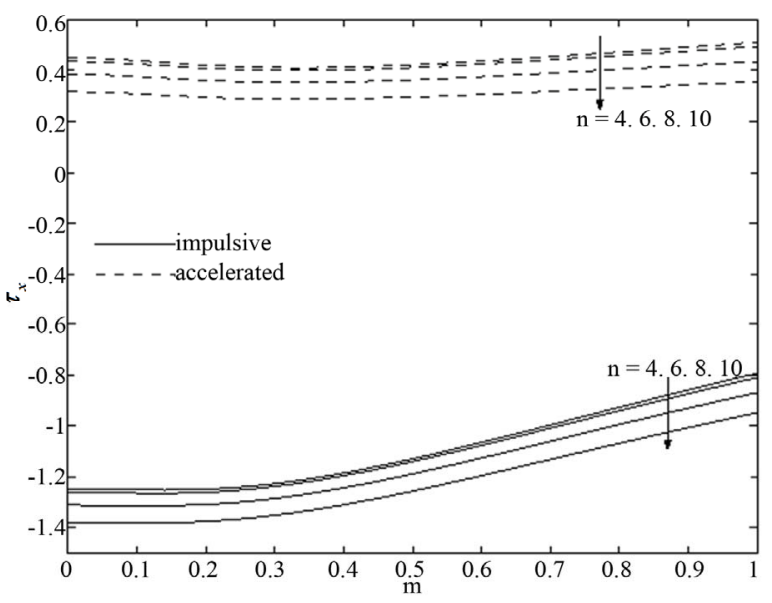

Figure 22. Shear stress $\tau_{x}$ for different $n$ when $R=2, \operatorname{Pr}=0.71, \mathrm{Gr}=5$ and $\tau=0.2$.

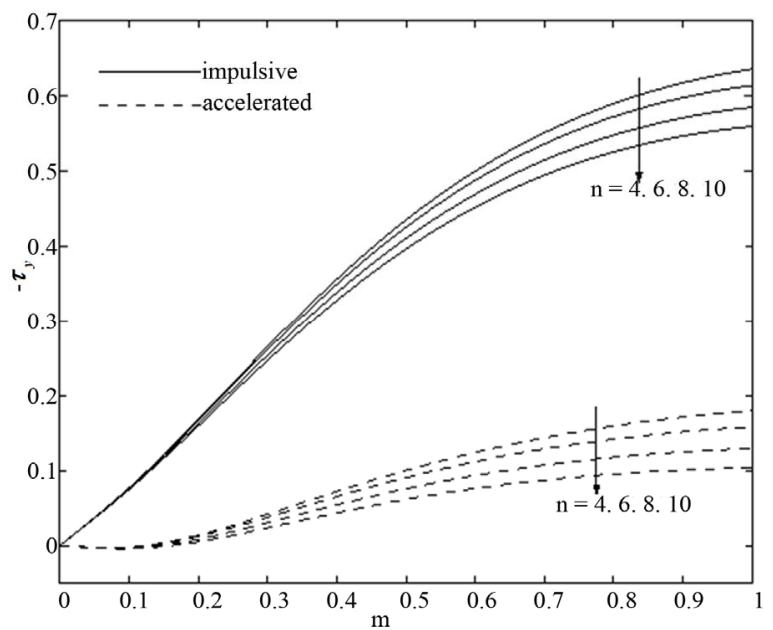

Figure 23. Shear stress $\tau_{y}$ for different $n$ when $R=2, \tau=0.2, \operatorname{Pr}=0.71$ and $\mathrm{Gr}=5$.

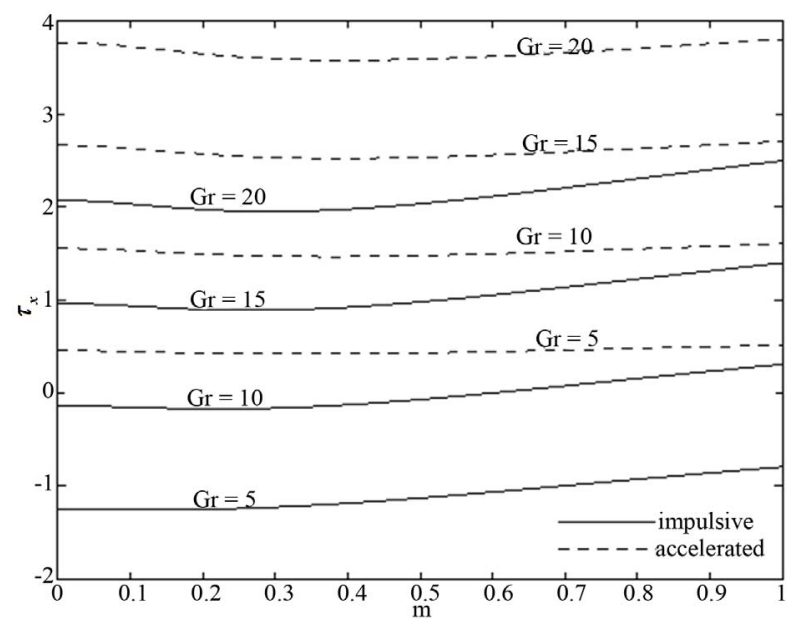

Figure 24. Shear stress $\tau_{x}$ for different $\mathrm{Gr}$ when $R=2, \operatorname{Pr}=0.71, n=2$ and $\tau=0.2$.

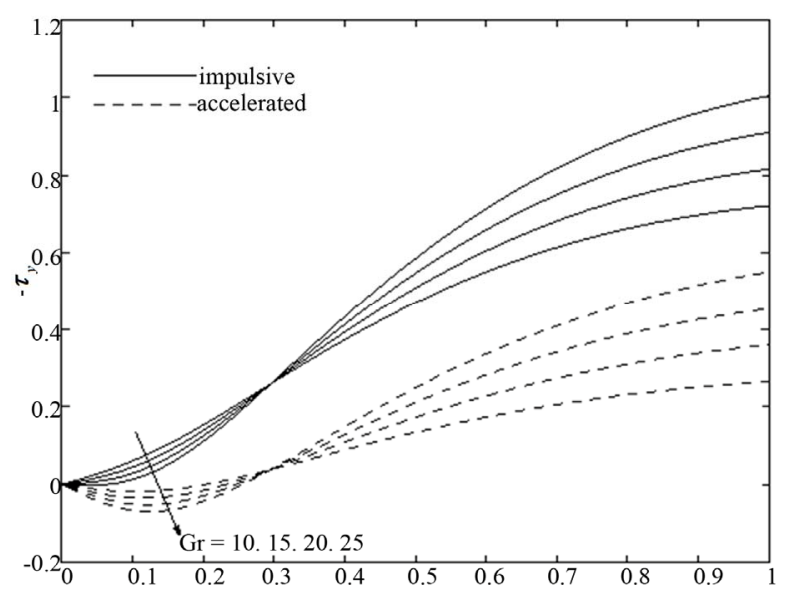

Figure 25. Shear stress $\tau_{y}$ for different $G r$ when $\tau=0.2, \operatorname{Pr}=0.71, n=2$ and $R=2$.

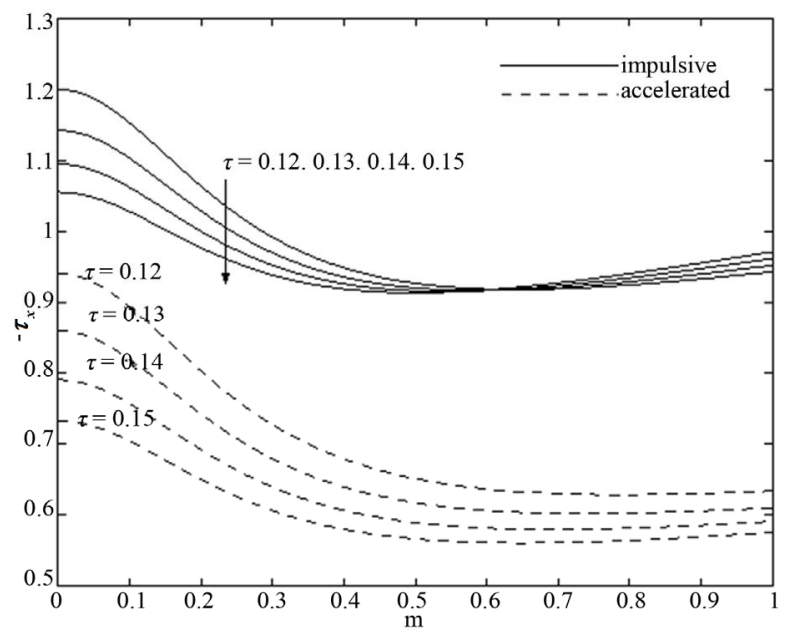

Figure 26. Shear stress $\tau_{x}$ for different time $\tau$ when $R=2, \operatorname{Pr}=0.71, n=2$ and $\mathrm{Gr}=5$.

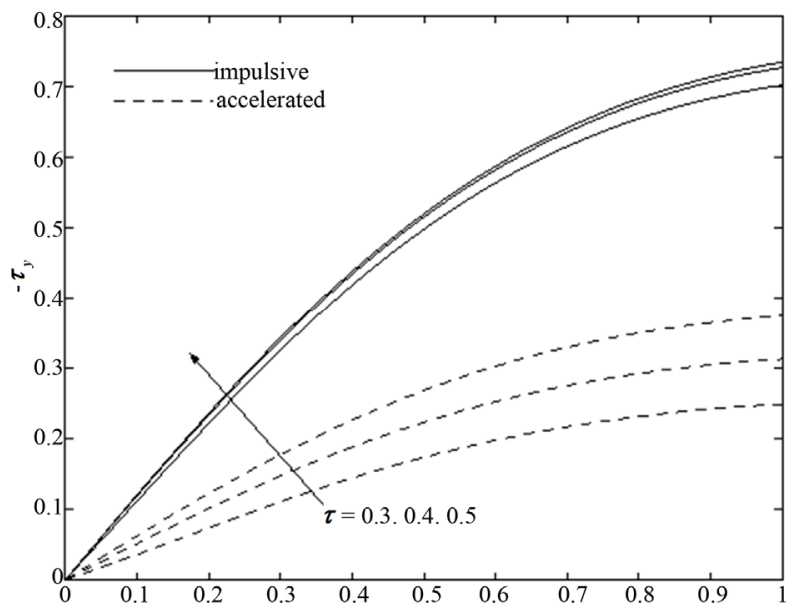

Figure 27. Shear stress $\tau_{y}$ for different time $\tau$ when $R=2, \operatorname{Pr}=0.71, n=2$ and $\mathrm{Gr}=5$. 
walls with an increase in radiation parameter $R$. It is seen from Figures $\mathbf{2 0}$ and $\mathbf{2 1}$ that for the impulsive and accelerated motions of one of the channel walls the shear stress $\tau_{x}$ and the magnitude of the shear stress $\tau_{y}$ increase with an increase of Prandtl number $\mathrm{Pr}$. Figures 22 and 23 show that the shear stress $\tau_{x}$ and the magnitude of the shear stress $\tau_{y}$ decrease with an increase in frequency parameter $n$ for the impulsive as well as accelerated motions of one of the channel walls. An increase of Grashof number $\mathrm{Gr}$ leads to increase in the shear stress $\tau_{x}$ while the magnitude of the shear stress $\tau_{y}$ decreases for $m \leq 0.3$ and increases for $m>0.3$ for both the impulsive and accelerated motions of one of the channel walls show in Figures 24 and 25. Figures 26 and 27 show that for both the impulsive and accelerated motions of one of the channel walls, the magnitude of the share stress $\tau_{x}$ decreases whereas the magnitude of the share stress $\tau_{y}$ increases with an increase in time $\tau$.

\section{Conclusion}

The combined effects of Hall current and radiation on the unsteady MHD free convective flow in a vertical channel with an oscillatory wall temperature have been studied. Radiation has a reterding influence on the fluid velocity components for both the impulsive as well as accelerated motions of one of the channel walls. Hall currents accelerates the fluid velecity components for the impulsive as well as accelerated motions of one of the channel walls. In the prence of radiation the fluid temperature $\theta$ decreases. Further, the shear stress $\tau_{x}$ and the absolute value of the shear stress $\tau_{y}$ at the wall $\eta=0$ decrease with an increase in radiation parameter $R$ for the impulsive as well as accelerated motions of one of the channel walls. The rate of heat transfers $-\theta^{\prime}(0, \tau)$ and $-\theta^{\prime}(1, \tau)$ increase with an increase in radiation parameter $R$.

\section{REFERENCES}

[1] H. Sato, "The Hall Effects in the Viscous Flow of Ionized Gas between Parallel Plates under Transverse Magnetic Field," Journal of Physical Society of Japan, Vol. 16, 1961, pp. 14-27. doi:10.1143/JPSJ.16.1427

[2] O. Miyatake and T. Fujii, "Free Convection Heat Transfer between Vertical Plates-One Plate Isothermally Heated and Other Thermally Insulated," Heat Transfer-Japanese Research, Vol. 1, 1972, pp. 30-38.

[3] H. Tanaka, O. Miyatake, T. Fujii and M. Fujii, "Natural Convection Heat Transfer between Vertical Parallel Plates-One Plate with a Uniform Heat Flux and the Other Thermally Insulated," Heat Transfer-Japanese Research, Vol. 2, 1973, pp. 25-33.

[4] P. S. Gupta and A. S. Gupta, "Radiation Effect on Hydromantic Convection in a Vertical Channel," Interna- tional Journal of Heat Mass Transfer, Vol. 17, No. 12, 1974, pp. 1437-1442. doi:10.1016/0017-9310(74)90053-2

[5] N. Datta and R. N. Jana, "Hall Effects on Hydromagnetic Convective Flow through a Channel with Conducting Walls," Internationa Journal of Engineering Science, Vol. 15, No. 9-10, 1977, pp. 561-567. doi:10.1016/0020-7225(77)90052-0

[6] A. R. Bestman and S. A. Adjepong, "Unsteady Hydromagnetic Free Convection Flow with Radiative Heat Transfer in a Rotating Fluid," Space Science, Vol. 143, No. 1, 1988, pp. 73-80. doi:10.1007/BF00636756

[7] H. M. Joshi, "Transient Effects in Natural Convection Cooling of Vertical Parallel Plates," International Communication Heat and Mass Transfer, Vol. 15, No. 2, 1988, pp. 227-238. doi:10.1016/0735-1933(88)90068-1

[8] A. K. Singh, "Natural Convection in Unsteady Couette Motion," Defense Science Journal, Vol. 38, No. 1, 1988, pp. 35-41.

[9] A. K. Singh, H. K. Gholami and V. M. Soundalgekar, "Transient Free Convection Flow between Two Vertical Parallel Plates," Heat and Mass Transfer, Vol. 31, No. 5, 1996, pp. 329-331. doi:10.1007/BF02184046

[10] B. K. Jha, "Natural Convection in Unsteady MHD Couette Flow," Heat and Mass Transfer, Vol. 37, No. 4-5, 2001, pp. 329-331. doi:10.1007/PL00013295

[11] M. Narahari, S. Sreenadh and V. M. Soundalgekar, "Transient Free Convection Flow between Long Vertical Parallel Plates with Constant Heat Flux at One Boundary," Journal of Thermophysics and Aeromechanics, Vol. 9, No. 2, 2002, pp. 287-293.

[12] B. K. Jha, A. K. Singh and H. S. Takhar, "Transient Free Convection Flow in a Vertical Channel Due to Symmetric Heating," International Journal of Applied Mechichal Engineering, Vol. 8, No. 3, 2003, pp. 497-502.

[13] A. K. Singh and T. Paul, "Transient Natural Convection between Two Vertical Walls Heated/Cooled Asymetrically," International Journal of Applied Mechanical Engineering, Vol. 11, No. 1, 2006, pp. 143-154.

[14] D. C. Sanyal and A. Adhikari, "Effects of Radiation on MHD Vertical Channel Flow," Bulletin of Calcutta Mathematical Society, Vol. 98, No. 5, 2006, pp. 487-497.

[15] P. Mebine, "Radiation Effects on MHD Couette Flow with Heat Transfer between Two Parallel Plates," Global Journal of Pure and Applied Mathematics, Vol. 3, No. 2, 2007, pp. 1-12.

[16] T. Grosan and I. Pop, "Thermal Radiation Effect on Fully Developed Mixed Convection Flow in a Vertical Channel," Technische Mechanik, Vol. 27, No. 1, 2007, pp. 37 47.

[17] M. Guria and R. N. Jana, "Hall Effects on the Hydromagnetic Convective Flow through a Rotating Channel under General Wall Conditions," Magnetohydrodynamics, Vol. 43, No. 3, 2007, pp. 287-300.

[18] B. K. Jha and A. O. Ajibade, "Unsteady Free Convective Couette Flow Of Heat Generating/Absorbing Fluid," International Journal of Energy and Technology, Vol. 2, No. 12, 2010, pp. 1-9.

[19] M. Narahari, "Effects of Thermal Radiation and Free 
Convection Currents on the Unsteady Couette Flow between Two Vertical Parallel Plates with Constant Heat Flux at One Boundary," WSEAS Transactions on Heat and Mass Transfer, Vol. 5, No. 1, 2010, pp. 21-30.

[20] U. S. Rajput and P. K. Sahu, "Transient Free Convection MHD Flow between Two Long Vertical Parallel Plates with Constant Temperature and Variable Mass Diffusion," International Journal of Mathematical Analysis, Vol. 5, No. 34, 2011, pp. 1665-6671.

[21] S. Das, B. C. Sarkar and R. N. Jana, "Radiation Effects on Free Convection MHD Couette Flow Started Exponentially with Variable Wall Temperature in Presence of Heat Generation," Open Journal of Fluid Dynamics, Vol. 2, No. 1, 2007, pp. 14-27.

[22] C. Mandal, S. Das and R. N. Jana, "Effect of Radiation on Transient Natural Convection Flow between Two Vertical Walls," International Journal of Applied Information Systems, Vol. 2, No. 2, 2012, pp. 49-56.

[23] S. Das, S. K. Guchhait and R. N. Jana, "Radiation Effects on Unsteady MHD Free Convective Couette Flow of Heat Generation/Absorbing Fluid," International Journal of Computer Applications, Vol. 39, No. 3, 2012, pp. 4251.

[24] B. C. Sarkar, S. Das and R. N. Jana, "Effects of Radiation on MHD Free Convective Couette Flow in a Rotating System," International Journal of Engineering Research and Application, Vol. 2, No. 4, 2012, pp. 2346-2359.

[25] B. C. Sarkar, S. Das and R. N. Jana, "Oscillatory MHD Free Convective Flow between Two Vertical Walls in a Rotating System," Advance in Applied Science Research, Vol. 3, No. 5, 2012, pp. 3311-3325.

[26] A. C. Cogley, W. C. Vincentine and S. E. Gilles, "A Differential Approximation for Radiative Transfer in a NonGray Gas Near Equilibrium," American Institute of Aeronautics and Astronautics Journal, Vol. 6, No. 3, 1968, pp. 551- 555.

[27] T. G. Cowling, "Magnetohydrodynamics," Interscience, New York, 1957. 